\title{
Numerical analysis of nonlinear oscillatory processes in columns at drilling oil and gas well
}

\author{
Lelya Khajiyeva ${ }^{1}$, Batyrgan Mardonov ${ }^{2}$, Sirozhiddin Amanov ${ }^{2}$ and Almatbek Kydyrbekuly ${ }^{1}$ \\ ${ }^{1}$ al-Farabi National University, 71, al-Farabi ave., 050040 Almaty, Kazakhstan \\ ${ }^{2}$ Tashkent Institute of Textile and Light Industry, 5, Shahjahan str, 100100 Tashkent, Uzbekistan
}

\begin{abstract}
The paper describes the behavior of the system of geometrically nonlinear drill columns under the action of dynamic loads. The authors used the finite element method to solve the boundary value problems. The forms of the beam in the transverse direction were expressed by cubic Hermite polynomials. It has been found out that nonlinearity of the column may substantially change the character of oscillatory processes in its transverse direction. The loss of stability may occur during slip kicking at high values of nonlinearity.
\end{abstract}

\section{Introduction}

Nonstationary oscillations of geometrically nonlinearly distributed systems make complicated problems in mechanics of deformed solid bodies and the theory of vibrations. Studying of such problems enabled scientists to reveal a number of poorly studied aspects including physically and geometrically non-linear processes accompanied by various types of drilling hazards (loss of column stability, pipe breaks, etc.) as well as wave and oscillatory processes in the elements of the dynamic drill system [1], [2].

The dynamic impact on the column can be produced by the engine torque and repeatedly varying loads during drilling with cutting bits, axial tensile loads from the drilling rig and compressive loads accompanied by vibrations during bore bit operations in the bottomhole, etc. The behavior of the column under the dynamic load depends on the dynamic characteristics of the column design, its frequencies and modes in longitudinal, transverse and torsional oscillations. It was found out that the impact of the torque on the behavior of the column is determined not by its magnitude but by a possible deviation of the column from the state of static equilibrium. In this case the twisted column loses its stability not as a result of static bending but as result of a flutter, when the energy supplied to the column is converted into the energy of transverse oscillations with the amplitude increasing in time.

Theoretically, the column must be considered as a non-linear mechanical system with an infinite number of degrees of freedom. However, there are no analytical methods that would enable specialists to study the dynamics of such a system and to evaluate the influence of vibrations on its strength and stability during drilling. In practice, the drill column usually loses its rectilinear form due to the loss of stability. Stability of drill columns was considered in [3], [4], where the influence of a concentrated force, the column weight, the internal and external pressure, centrifugal forces, deformation properties of the pipe material and other factors on column stability was studied. Construction and specificity of operation of drilling columns make it possible to represent them as long heavy stretched-compressed tubular rods experiencing constant action of longitudinal and transverse loads. In the study of dynamic stability of drill columns under the action of dynamic loads, new effects caused by the nature of loads arise [3]. Such effects, in particular, include fast loading of the column by the axial compressing load. If the rate of increase in the pressing force is rather high, the cross section of the drill column does not have time to move in the direction normal to the axis of the column. Because of this, the compressive force can reach the first critical value and even exceed it considerably earlier than bending reaches significant values. As in this dynamic process the compressive force can exceed not only the first but also higher critical values, higher forms of stability loss can be expected. This feature of the dynamic loading process is important because it causes a significant increase in the carrying capacity of the column. If the rate of increase of the pressing force is much smaller than the speed of propagation of longitudinal waves in the drill column, the time interval during which the wave front reaches the lower end of the column is small as compared to this time. In such a case we can assume that the process is quasistationary and the compressive force at any given moment of time in all sections of the column changes immediately. If the compressive load is applied during a short period of time, which corresponds to an impact plunge of the column, it is necessary to follow the process of force transfer along the length of the column, 
and thus to take into account the inertia forces of the column elements corresponding to the longitudinal movement.

To obtain quantitative solutions of nonlinear problems in the elasticity theory it is often necessary to use numerical methods. One of such approaches based on the idea of piecewise approximation of continuous fields is the finite element method. The simplicity and similarity of this method make it a convenient tool for solving a wide class of nonlinear problems [5]-[7].

\section{Mathematical model}

Let us consider vibrations of drilling rods (columns) under the action of time-varying compression loads. It is assumed that the column is a heavy rod. The deformed state of the drill column is assumed to be geometrically nonlinear, i.e. finite deformations are considered to be acceptable. Let us determine the potential energy of bending and longitudinal displacement of the rod in the case of non-linear deformation $U$ [3], [8], [9]:

$$
\begin{aligned}
& U=\frac{E I}{2} \int_{0}^{l}\left[\frac{\partial^{2}\left(v_{1}-v_{0}\right)}{\partial x^{2}}\right]^{2} d x+ \\
& +\int_{0}^{l}\left[\left(\frac{\partial u}{\partial x}\right)^{2}+\left(\frac{\partial u}{\partial x}\right)\left(\frac{\partial v_{1}}{\partial x}\right)^{2}+\frac{1}{2}\left(\frac{\partial v_{1}}{\partial x}\right)^{4}\right] d x
\end{aligned}
$$

the work $A_{1}$ of the external force $P$ in the vertical displacement, the work $A_{2}$ of the weight of the rod when the rod deviates from its equilibrium position are:

$$
\begin{gathered}
A_{1}=\frac{P}{2} \int_{0}^{l}\left[\left(\frac{\partial v_{1}}{\partial x}\right)^{2}-\left(\frac{\partial v_{0}}{\partial x}\right)^{2}\right] d x+\frac{P l}{E F}, \\
A_{2}=-\frac{q}{2} \int_{0}^{l}[x+l(i-1)]\left(\frac{\partial v_{i}}{\partial x}\right)^{2} d x
\end{gathered}
$$

The kinetic energy $T$ is

$$
T=\frac{q F}{2 g} \int_{0}^{l}\left\{\left(\frac{\partial v_{1}}{\partial t}\right)^{2}+\left(\frac{\partial u}{\partial t}\right)^{2}\right\} d x,
$$

where $v_{0}(x)$ is the initial form of bending, $v_{1}=v_{1}(x, t)$ is the total bending of the rod, $u=u(x, t)$ is the longitudinal displacement of cross-sections of the rod, $E$ is the Young module for the rod material, $l, F, I$ are the length, the cross-sectional area and the moment of inertia, $q$ is the linear weight of the rod.

The total potential energy is: $Э=U-A_{1}-A_{2}$ [3]. Let us define the Lagrangian as $L=T-\ni$. Taking $q=q(t)$ as a generalized coordinate, we can write the Lagrange equation of the second type:

$$
\frac{d}{d t}\left(\frac{\partial L}{\partial \dot{q}}\right)-\frac{\partial L}{\partial q}=0 .
$$

It means that in the energy method it is necessary to determine the expressions for all potentials and the kinetic energy in terms of generalized coordinates. The deflections of the beam $v_{0}=v_{0}(x)$ and $v_{1}=v_{1}(x, t)$ and the longitudinal displacement $u=u(x, t)$ can be represented by arbitrary functions satisfying corresponding initial and boundary conditions.

\section{Discretization of model}

Let us consider the following boundary value problem :

$$
\begin{gathered}
v_{1}(x, t)=0, \frac{\partial v_{1}(x, t)}{\partial x}=0, \frac{\partial u}{\partial x}=\frac{P(t)}{E F} \text { for } x=0 \\
v_{1}(x, t)=0, \frac{\partial v_{1}(x, t)}{\partial x}=0, u=u_{0}(t) \text { for } x=l
\end{gathered}
$$

Finite element method.is used to solve of boundary value problem. The length of the drilling rod is divided into $n$ finite elements $(n+1$ nodes) of the same length $a$. The form of the beam in the transverse direction is expressed by the cubic Hermite polynomials:

$$
\begin{gathered}
\Im_{1}=1-2 \xi^{2}+2 \xi^{3}, Э_{2}=a\left(\xi-2 \xi^{2}+\xi^{3}\right), \\
Э_{3}=3 \xi^{2}-2 \xi^{3}, Э_{4}=a\left(-\xi^{2}+\xi^{3}\right), \xi=x / a .
\end{gathered}
$$

The longitudinal displacement of each element can be expressed in terms of quadratic functions: $N_{1}=1-3 \xi+2 \xi^{2}, N_{2}=2 \xi^{2}-\xi$.

Therefore, for an arbitrary element the bending of the beam can be written as $v=\ni_{1} q_{i}+\ni_{2} q_{i+1}+\ni_{3} q_{j}+Э_{4} q_{j+1}$, where $q_{i}, q_{j}$ are deflections, $q_{i+1}, q_{j+1}$ are angles of rotation in the nodes of elements. The total number of unknowns for the chosen $n$ elements is $2 n+2$.

The longitudinal displacement of cross-sections of the element is expressed as $u=N_{1} r_{i}+N_{2} r_{j}$, where $r_{i}, r_{j}$ are longitudinal displacement in the nodes of the element. The number of unknown displacements of nodes is equal to $n+1$. Thus, in the general case the chosen system of finite elements has $3 n+3$ degrees of freedom, corresponding to bendings $v=q_{i}, v=q_{j}$, angles of rotation $v^{\prime}=q_{i+1} v^{\prime}=q_{j+1}$ and longitudinal displacements $u=r_{i}, u=r_{j}$. Further, these coordinates are called generalized. Taking into account the boundary conditions (6) - (7), the number of unknowns becomes equal to $k=3 n+3-6=3(n-1) \quad(n \geq 2)$. Let us introduce the following notations: $v_{i, i+1}$ is the beam bending in the element located between $i$ and $i+1$ nodes and $u_{i, i+1}$ are longitudinal displacements. According to the boundary conditions (6) - (7) $v_{i, i+1}$ can be expressed as:

$$
\begin{gathered}
v_{1,2}=Э_{3} q_{3}+Э_{4} q_{4}, \\
v_{2,3}=Э_{1} q_{3}+Э_{2} q_{4}+Э_{3} q_{5}+Э_{4} q_{6},
\end{gathered}
$$




$$
\begin{gathered}
v_{3,4}=Э_{1} q_{5}+Э_{2} q_{6}+Э_{3} q_{7}+Э_{4} q_{8} \\
\ldots \ldots \\
v_{i, i+1}=Э_{1} q_{2 i-1}+Э_{2} q_{2 i}+Э_{3} q_{2 i+1}+\ni_{4} q_{2 i+2}(i=2, n-1), \\
\ldots \ldots \ldots \\
v_{n-1, n}=\left(1-3 \xi^{2}+2 \xi^{3}\right) q_{2 n-3}+Э_{2} q_{2 n-2}
\end{gathered}
$$

For longitudinal displacements and $u_{i, i+1}$ is obtained:

$$
\begin{gathered}
u_{1,2}=\left(1-4 \xi^{2}\right) r_{1}+N_{2} \frac{P a}{E F}, \\
u_{2,3}=\left(-3 r_{1}+\frac{P a}{E F}\right) N_{1}+N_{2} r_{3}, \\
u_{3,4}=N_{1} r_{3}+N_{2} r_{4}, \\
\ldots \ldots, \\
u_{i, i+1}=N_{1} r_{i}+N_{2} r_{i+1}, \quad(i=3 . . n-1) \\
\ldots \ldots \ldots . \\
u_{n, n+1}=N_{1} r_{n}+N_{2} u_{0} .
\end{gathered}
$$

Substituting expressions $v=v(\xi, t)$ and $u(\xi, t)$ into formulae (1)-(3), determine the potential energy of deformation, the potential load $\mathrm{P}$, the potential weight force and kinetic energy through the Hermite polynomials.

In case of the dynamic drill system with concentrated masses (clutches), the energy of the beam and concentrated masses $m_{i}$ can be expressed as:

$$
T_{i}=\frac{q F}{2 g} \int_{0}^{l}\left\{\left(\frac{\partial v_{i}}{\partial t}\right)^{2}+\left(\frac{\partial u_{i}}{\partial t}\right)^{2}\right\} d x+\frac{1}{2} m_{i}\left(\frac{\partial u_{i}(0, t)}{\partial t}\right)^{2}
$$

where $u_{i}=u_{i}(t)$ is the displacement of the $i-$ th mass, and $u_{n}(l, t)=u_{0}(t)\left(u_{0}(t)\right.$ is the displacement of the lower end of the $n$-th beam).

The sum of potentials of the beam weight, compression $P$ and concentrated masses is calculated by the formula

$$
V_{i}=-\frac{q}{2} \int_{0}^{l}[x+l(i-1)]\left(\frac{\partial v_{i}}{\partial x}\right)^{2} d x-m_{i} g u_{i}(0, t) .
$$

To determine the longitudinal displacement of the rod we will use Kirchhoff's hypothesis [12], according to which the average elongation $\varepsilon_{i x}$ of each drilling rod does not dependent on coordinates and depends only on time. Now we obtain the following expression for the deformation [10]:

$$
\begin{aligned}
& \varepsilon_{i x}=\frac{\partial u_{i}}{\partial x}+\frac{1}{2}\left(\frac{\partial v_{i}}{\partial x}\right)^{2} \\
& \varepsilon_{i x}=\frac{1}{l}\left[u_{i}(l, t)-u_{i}(0, t)+\frac{1}{2} \int_{0}^{l}\left(\frac{\partial v_{i}}{\partial x}\right)^{2} d x\right]
\end{aligned}
$$

The longitudinal displacement of the $i$ - th beam is expressed as

$$
u_{i}=r_{i-1} \frac{l-x}{l}+r_{i} \frac{x}{l} \quad(i=2 \ldots n)
$$

where $\quad r_{i-1}=r_{i-1}(t)$ and $r_{i}=r_{i}(t)$ are displacements of the initial and final cross-sections of the $i$ - th pipe (stalk), with $r_{n}=u_{0}(t)$. On the boundaries of pipe connections the conditions of continuity of efforts must be fulfilled:

$$
E F \frac{d u_{i}}{d x}=E F \frac{d u_{i+1}}{d x}=k\left[u_{i+1}(0)-u_{i}(l)\right], E F \frac{d u_{1}}{d x}=-P_{0} .
$$

Taking into account Kirchhoff's hypothesis the expressions for potential and kinetic energy of the $i$-th beam with regard to (21) and (23) are written as:

$$
\begin{gathered}
U_{1}=\frac{E I}{2} \int_{0}^{l}\left(\frac{\partial^{2} v_{i}}{\partial x^{2}}\right)^{2} d x+\frac{E F}{2 l}\left[-\frac{P_{0} l}{E F}+\frac{1}{2} \int_{0}^{l}\left(\frac{\partial v_{i}}{\partial x}\right)^{2} d x\right]^{2}, \\
U_{i}=\frac{E I}{2} \int_{0}^{l}\left(\frac{\partial^{2} v_{i}}{\partial x^{2}}\right)^{2} d x+\frac{E F}{2 l}\left[r_{i}-r_{i-1}+\frac{1}{2} \int_{0}^{l}\left(\frac{\partial v_{i}}{\partial x}\right)^{2} d x\right]^{2}, \\
T_{i}=\frac{q F}{2 g} \int_{0}^{l}\left(\frac{\partial v_{i}}{\partial t}\right)^{2} d x+\frac{1}{2} m_{i} \dot{r}_{i-1}^{2}, \quad\left(i=1 \ldots n, \dot{r}_{0}=0\right) .
\end{gathered}
$$

Substituting expressions (24) - (26) into (5), we obtain a system of $3 n-3$ nonlinear equations, which can be used to determine generalized coordinates $q_{i}=q_{i}(t), r_{i}=r_{i}(t)$. For example, for $n=3$ we obtain the following system:

$$
\begin{gathered}
\quad \rho l^{2}\left(a_{11} \ddot{\bar{q}}_{1}+a_{13} \ddot{\bar{q}}_{3}+a_{14} \ddot{q}_{4}\right)= \\
-\left(b_{11} \bar{q}_{1}+b_{12} q_{2}+b_{13} \bar{q}_{3}+b_{14} q_{4}\right)+F_{1}\left(\bar{q}_{1}, q_{2}, \bar{q}_{3}, q_{4}, \bar{r}_{1}, \bar{r}_{2}, t\right), \\
\quad \rho l^{2}\left(a_{22} \ddot{q}_{2}+a_{23} \ddot{\bar{q}}_{3}+a_{24} \ddot{q}_{4}\right)= \\
-\left(b_{12} \bar{q}_{1}+b_{22} q_{2}+b_{23} \bar{q}_{3}+b_{24} q_{4}\right)+F_{2}\left(\bar{q}_{1}, q_{2}, \bar{q}_{3}, q_{4}, \bar{r}_{1}, \bar{r}_{2}, t\right), \\
\rho l^{2}\left(a_{13} \ddot{q}_{1}+a_{23} \ddot{\bar{q}}_{2}+a_{33} \ddot{q}_{3}\right)= \\
-\left(b_{13} \bar{q}_{1}+b_{23} q_{2}+b_{33} \bar{q}_{3}+b_{34} q_{4}\right)+F_{3}\left(\bar{q}_{1}, q_{2}, \bar{q}_{3}, q_{4}, \bar{r}_{1}, \bar{r}_{2}, t\right), \\
\qquad \rho l^{2}\left(a_{14} \ddot{\bar{q}}_{1}+a_{24} \ddot{q}_{2}+a_{44} \ddot{q}_{4}\right)= \\
-\left(b_{14} \bar{q}_{1}+b_{24} q_{2}+b_{34} \bar{q}_{3}+b_{44} q_{4}\right)+F_{4}\left(\bar{q}_{1}, q_{2}, \bar{q}_{3}, q_{4}, \bar{r}_{1}, \bar{r}_{2}, t\right), \\
\quad m_{1} l \ddot{\bar{r}_{1}}=-E F\left(\bar{r}_{1}-\bar{r}_{2}\right)+F_{5}\left(\bar{q}_{1}, q_{2}, \bar{q}_{3}, q_{4}, \bar{r}_{1}, \bar{r}_{2}, t\right), \\
m_{2} \mid \ddot{\bar{r}}=-E F\left(2 \bar{r}_{2}-\bar{r}_{1}-\bar{u}_{0}\right)+F_{6}\left(\bar{q}_{1}, q_{2}, \bar{q}_{3}, q_{4}, \bar{r}_{1}, \bar{r}_{2}, t\right), \\
\text { where } \rho=q / g, \bar{q}_{1}=q_{1} / l, \bar{q}_{3}=q_{3} / l, \\
\bar{r}_{1}=r_{1} / l, \quad \bar{r}_{2}=r_{2} / l, \quad \bar{u}_{0}=u_{0} / l, \\
a_{11}=52 / 35, a_{13}=9 / 35, a_{14}=-13 / 210, \\
a_{22}=4 / 105, a_{23}=13 / 210, a_{24}=-1 / 70, \\
a_{33}=52 / 35, a_{44}=4 / 105, \\
b_{11}=48 E J / l^{2}+24 q l / 5+P_{0} / 5, b_{12}=2 q l / 5, \\
b_{13}=-24 E J / l^{2}-18 q l / 5-12 P_{0} / 5, \\
b_{14}=12 E J / l^{2}+q l / 5+P_{0} / 5, \\
b_{22}=16 E J / l^{2}+8 q l / 15+8 P_{0} / 15, \\
b_{23}=-12 E J / l^{2}-2 q l / 5-P_{0} / 5,
\end{gathered}
$$


$b_{24}=4 E J / l^{2}-q l / 10-P_{0} / 5$,

$b_{33}=48 E J / l^{2}+48 q l / 5+24 P_{0} / 5$,

$b_{34}=2 q l / 5, b_{44}=16 E J / l^{2}+16 q l / 15+8 P_{0} / 15$,

$F_{i}$ are nonlinear components of derivatives $\frac{\partial U_{i}}{\partial q_{i}}, \frac{\partial U_{i}}{\partial r_{1}}$, $\frac{\partial U_{i}}{\partial r_{2}}(i=1 \ldots 4)$

\section{Numerical results}

In calculations the following values $n=3, k=1 / \mathcal{M}$, $v_{0}=2 \mathrm{M} / \mathrm{c}, l=1000_{\mathrm{M}}, D_{1}=140_{\mathrm{MM}}, D_{2}=128 \mathrm{m \mu}$, $E=2 \cdot 10^{5} \mathrm{MPa}, \rho=\rho_{0} F, \rho_{0}=7000 \kappa 2 / \mathrm{M}^{3}, m_{1}=40 \kappa 2$, $m_{2}=40 \kappa 2, P_{0}=485 \mathrm{H}$ were used.

Figure 1 shows the curves of displacement of the bottom part of the column obtained using the formula $u_{0}=\frac{\ln \left(k v_{0} t+1\right)}{k}$, and longitudinal displacement of the concentrated mass $m_{2}$ in time.

It has been found out that the concentrated mass and the lower part of the column make not only the translational movement but also the oscillatory motion. The calculations show that concentrated masses produce a negligible impact on the longitudinal displacements of ring junctions.
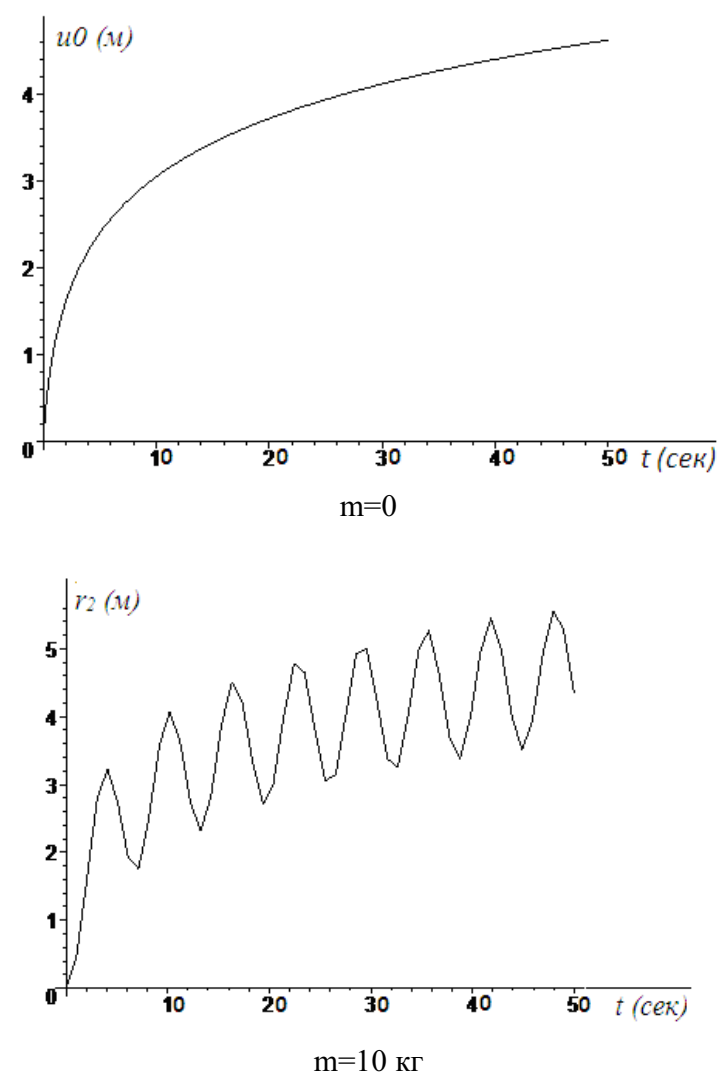

Figure 1. Displacements of the bottom of the column $u_{0}(t)(\mathrm{m})$ and the concentrated mass $r_{2}(t)(\mathrm{m})$ as a function of time $t(s)$.
Fig. 2 and 3 show the dependence of the transverse displacements of junctions $q_{1}$ and $q_{3}$ on time for various values of masses $m_{1}=m_{2}=m$.
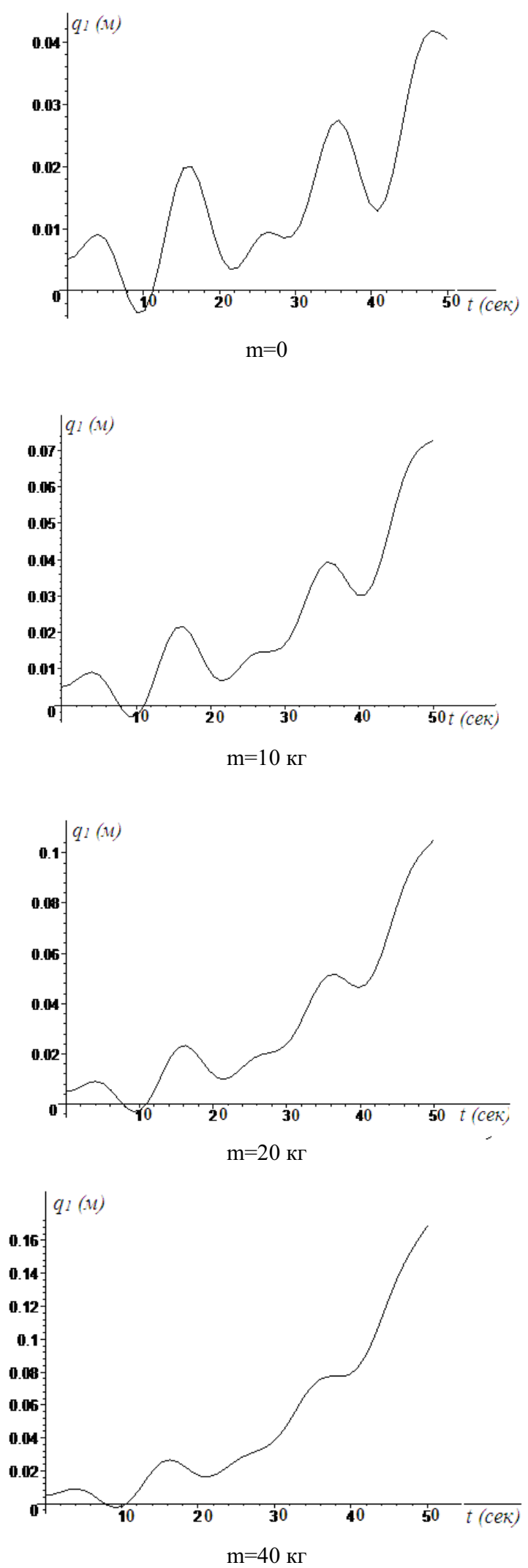

Figure 2. Transverse displacement of the first junction $q_{1}(\mathrm{~m})$ as a function of time $t$ (s) for various mass values $m(\mathrm{~kg})$. 

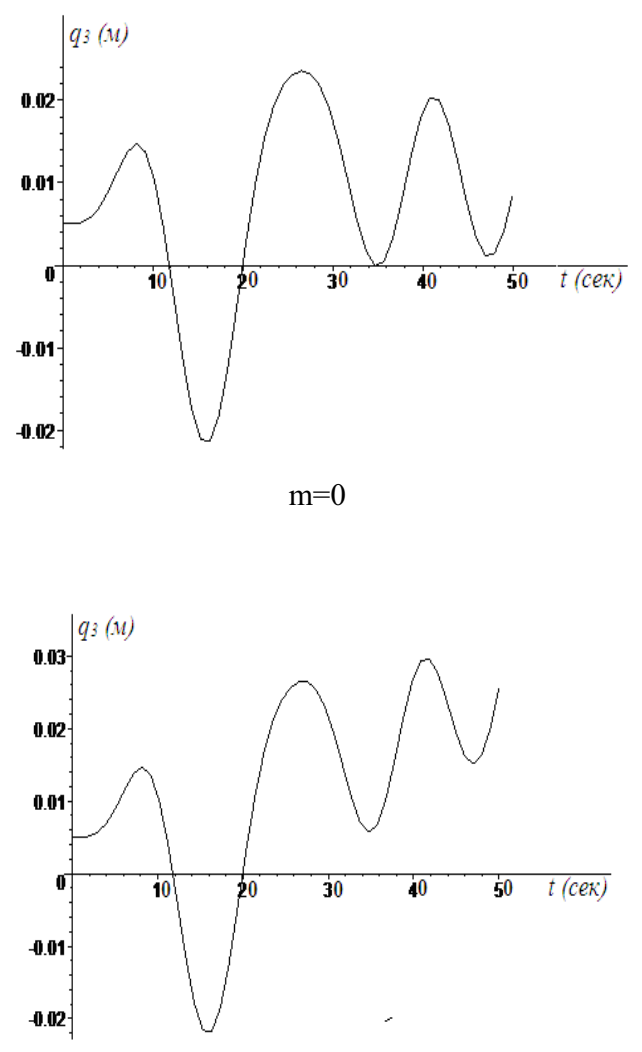

$\mathrm{m}=10$ кг

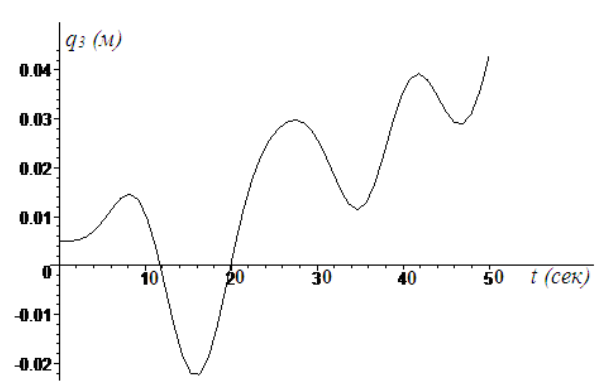

$\mathrm{m}=20$ кг

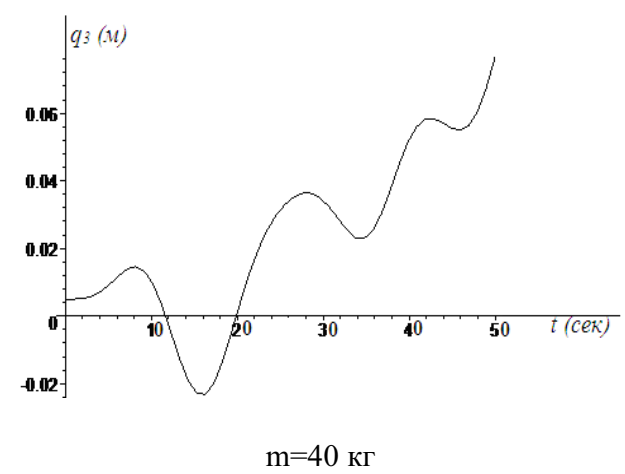

Figure 3. Transverse displacement of the second junction $q_{1}(\mathrm{~m})$ as a function of time $t(\mathrm{~s})$ for various masses $m(\mathrm{~kg})$.

The analysis of the curves shows that the presence of concentrated masses in junctions of column parts causes changes in frequencies and an increase in the amplitude of their vibrations. This indicates a change in the type of transverse vibrations in sleeve joints between stalks of the column when masses of sleeve joints are taken into account.

\section{Conclusion}

An analysis of the dynamics of the drill column under the action of variable axial forces has shown that the presence of concentrated masses practically does not affect the amplitude of longitudinal vibrations. However, they may have a significant impact on the transverse vibrations of the drilling rod. They cause an asymmetric distortion in the bending of the drilling rod, which means that it may lose its stability.

The duality of the deformed state of the drilling rod is explained by its geometric nonlinearity. The loss of stability may occur during slip kicking at high values of nonlinearity. However, the probability of transition to an unstable state in the initial moments of time is reduced.

\section{Acknowledgment}

The research work was partially supported by the scientific grant from the Ministry of Education and Science of the Republic of Kazakhstan (Project $\mathrm{N}^{0}$ ГР112РК01496).

\section{References}

1. B.M. Mardonov, I. Mardanova, Poisk, Series of nat. and techn. sci. 1, 217-220 (2001)

2. B.M. Mardonov, A.B. Barayev, N.M. Akhmetov, Applied problems of mechanics of drilling oil and gas wells ( Alem, Shymkent, 2013)

3. A.S. Volmir, Stability of elastic systems (Fizmatgiz, Moscow, 1963)

4. I.L. Barsky, T.V. Bredikhina, I.N. Genkina, M.A. Tchaikovskaya, N.E. Shurova, Papers of VNIIBT, 60, 38-43 (1985)

5. O. Zenkevich, Finite Element Method in technology (Mir, Moscow, 1975)

6. I.F. Obraztsov, L.M. Savelyev, H.S. Khazanov H.S. FEM in problems of structural mechanics of aircrafts (High School, Moscow, 1985)

7. J. Oden, Finite elements in nonlinear continuum mechanics (Mir, Moscow, 1976)

8. M.J. Turner, E.H. Dill, H.C. Martin, R.J. Melosh, J.Aerospace Sce. 27, 97-102 (1960)

9. R.W. Glough, 2d Conf. Electronic Compilation, J.Struct. Div. ASCE, 345-378 (1960)

10. V.V. Novozhilov, Fundamentals of nonlinear elasticity theory ( OGIZ, M-L., 1948)

11. L.A. Khajiyeva, Models of nonlinear deformable systems. Practical application (Kazak universiteti, Almaty, 2004)

12. G. Kauderer, Nonlinear mechanics ( M. Publ. house. Foreign, IL, Moscow, 1960) 